\title{
Hemodynamic and Neuroendocrinological Responses to Artificial Gravity
}

\author{
Stefan Schneider ${ }^{1,2,3}$, Vanja Sebastian Zander ${ }^{1,2}$, Tobias Vogt ${ }^{1,2}$, Vera Abeln ${ }^{1,2}$, Heiko K. Strüder ${ }^{1}$, \\ Amrei Jacubowski ${ }^{1}$, Heather Carnahan ${ }^{4}$, and Petra Wollseiffen ${ }^{1}$ \\ ${ }^{1}$ Institute of Movement and Neurosciences, German Sport University Cologne, Germany; ${ }^{2}$ Centre for Health and \\ Integrative Physiology in Space, German Sport University Cologne, Germany; ${ }^{3}$ Faculty of Science, Health, \\ Education and Engineering, University of Sunshine Coast, Maroochydore, Queensland, Australia; ${ }^{4}$ Offshore \\ Safety and Survival Centre, Marine Institute, Memorial University of Newfoundland, Canada
}

\begin{abstract}
The aim of this study was to determine the hemodynamic and neuroendocrinological responses to different levels and protocols of artificial gravity, especially in comparison to what is expected during a moderate bout of exercise. Ten male participants were exposed to artificial gravity using two different protocols: the first was a centrifugation protocol that consisted of a constant phase of $2 \mathrm{Gz}$ for 30 minutes, and the second consisted of an intermittent phase of $2 \mathrm{Gz}$ for two minutes, separated by resting periods for three minutes in successive order. Near infrared spectroscopy (oxyhemoglobin and deoxyhemoglobin) at the prefrontal cortex, Musculus biceps brachii, and Musculus gastrocnemius, as well as heart rate and blood pressure were recorded before, during, and after exposure to artificial gravity. In order to determine effects of artificial gravity on neuroendocrinological parameters (brain-derived
\end{abstract}

Key words: $\quad$ Exercise Countermeasure; NIRS (Near Infrared Spectroscopy); Hemodynamics; Neuroendocrinology; Artificial Gravity; Spaceflight

Correspondence to: Prof. Dr. Stefan Schneider Institute of Movement and Neurosciences German Sport University Am Sportpark Müngersdorf 6 D-50933 Cologne, Germany Telephone: +49 (0) 22149827520 Fax: +49 (0) 2214973454 E-Mail: schneider@dshs-koeln.de neurotrophic factor, vascular endothelial growth factor, and insulin-like growth factor 1), blood samples were taken before and after centrifugation. During the application of artificial gravity the concentration of oxyhemoglobin decreased significantly and the concentration of deoxyhemoglobin increased significantly in the prefrontal cortex and the Musculus biceps brachii muscle. Participants exposed to the continuous artificial gravity profile experienced peripheral pooling of blood. No changes were observed for brain-derived neurotrophic factor, vascular endothelial growth factor, or insulin-like growth factor 1 . Intermittent application of artificial gravity may represent a better-tolerated presentation for participants as hemodynamic values normalize during resting periods. During both protocols, heart rate and arterial blood pressure remained far below what is experienced during moderate physical activity.

\section{INTRODUCTION}

Astronauts experience arterial blood pressure $(\mathrm{ABP})$ variations, decrements in bone density, cardiovascular degradation, and neurocognitive deconditioning during long-term space missions (Schneider, 2016). In order to prevent these physiological changes, efficient countermeasures applicable during human spaceflight are needed (Clement et al., 2015; Richter et al., 2017; Tanaka et al., 2017; Linnarsson et al., 2015; Waki et al., 2005). Various countermeasures have been investigated 
during space missions in recent years. Lower body negative pressure devices, exercises with bungee cords, modified bicycles, self-propelled centrifuge devices, and fixed treadmills have been assessed and used to counteract negative physiological responses (Clement and Bukley, 2007). However, all countermeasures mentioned require a significant amount of crew time and still appear to be inefficient in counteracting the degradation of physiological structures. The application of artificial gravity (AG) generated by, for example, a short arm human centrifuge (SAHC) is under intense scientific investigation (Chouker et al., 2013; Clement and Pavy-Le Traon, 2004; Dern et al., 2014; Evans et al., 2015; Goswami et al., 2015; Linnarsson et al., 2015; Zhang et al., 2017). Centrifugation in a sitting or supine position generates a centrifugal force, which simulates the gravity-induced physiological effects, similar to standing. In addition to the implementation of SAHC in microgravity environments, these devices could be installed on planetary destinations, such as Mars or the Moon, where gravity is only a fraction of Earth's gravity. Additionally, the application of AG generated by a SAHC is also thought to be beneficial in the fields of competitive sports (Barr et al., 2015), rehabilitation, and therapy for immobilized persons (Pavy-Le Traon et al., 2007). Besides possible positive effects on the musculoskeletal system, there are also possible beneficial effects of AG on the cardiovascular system (Migeotte et al., 2009), which are considered to be as effective as exercise to counteract physiological deconditioning during spaceflight (Clement and Bukley, 2007).

The primary aim of this study was to determine the effects of various $A G$ protocols on the cardiovascular system in order to determine (1) if AG at a tolerable level stimulates an increase in ABP and heart rate (HR), similar to what would be expected during a moderate bout of exercise, and (2) if different protocols, intermittent $A G$ vs. continuous $A G$, might have a different impact on the above mentioned parameters.

The activation of the cardiovascular system has a modulating effect on the release of neurotrophic factors such as brain-derived neurotrophic factor (BDNF), vascular endothelial growth factor (VEGF), and insulinlike growth factor 1 (IGF-1) (Knaepen et al., 2010; Rojas Vega et al., 2010; Schiffer et al., 2009), which all play key roles in the signaling pathways of neurogenesis (BDNF), synaptogenesis (BDNF, IGF-1), and angiogenesis (IGF-1, VEGF) (Lista and Sorrentino, 2010). The second aim of this study was to determine the effect of AG on the release of these proteins. Based on the assumption of cardiovascular system activation, we hypothesized that BDNF, VEGF, and IGF-1 would increase after exposure to AG.

The third aim of this study was to further investigate blood volume redistribution following different levels of AG (Schneider et al., 2013). It was hypothesized that AG generated by a SAHC would decrease cerebral oxygenation, increase cerebral deoxygenation, and increase peripheral intravascular pooling, which can be assessed using near infrared spectroscopy (NIRS). Although alterations in hemodynamics have been assessed in previous studies, the relationship between the gradient in the centrifugal force generated by a SAHC, and oxygenation of various tissues, such as the brain, during continuous and intermittent application of AG has been under-investigated.

\section{MATERIALS AND METHODS}

\section{Participants and Procedures}

Ten healthy males $(29.57 \pm 6.61$ years, $183.21 \pm 5.11 \mathrm{~cm}, 82.14 \pm 7.24 \mathrm{~kg})$ participated in this study. Participants had a varied experience with AG and hypergravity. All participants underwent a preliminary medical screening performed by medical personnel at the Institute of Aerospace Medicine/German Aerospace Centre (DLR), Cologne, Germany, and all gave their written consent to participate in the study. None of the participants reported taking any medication during the study. Before the centrifugation at the SAHC, the participants were informed about the experimental protocol and the risks of centrifugation (nausea, syncope, tachycardia, dizziness, etc.). All experimental procedures were performed according to national legislation and the Declaration of Helsinki; methods were performed in accordance with relevant regulations and guidelines and were approved by the Medical Council - North Rhine (Ärztekammer Nordrhein, Düsseldorf, Germany).

\section{Experimental protocol procedure}

The study was conducted at the Institute of Aerospace Medicine at the German Aerospace Centre in Cologne, Germany, which granted access to the SAHC, which is owned and operated by the European Space Agency (ESA). 
On experimental days, participants arrived two hours before centrifugation. Two different AG protocols were performed with a time lag of at least 48 hours between each. Participants were exposed to $A G$ in a supine position and exposure to the two protocols was randomized. To avoid influencing hemodynamic parameters, participants were instructed not to flex their leg muscles during AG. The Gz-load was measured at foot level. The "continuous" AG protocol is illustrated in Figure 1A; it commenced with a phase of $1 \mathrm{Gz}, 2 \mathrm{Gz}$, and $3 \mathrm{Gz}$ for $120 \mathrm{~s}$ each (incremental phase 1), followed by a continuous phase of $2 \mathrm{Gz}$ for $30 \mathrm{~min}$, and ended with a phase of $1 \mathrm{Gz}, 2 \mathrm{Gz}$, and $3 \mathrm{Gz}$ for $120 \mathrm{~s}$ each (incremental phase 2; Figure 1A). For detailed analysis, the phase of continuous Gz-load was divided into six time segments. In contrast, the intermittent protocol is illustrated in Figure 1B, and included a phase of intermittent Gz-load of $2 \mathrm{Gz}$ and approximately $0.0279 \mathrm{Gz}$ (baseline rotation of 5 rounds per minute (RPM)) for 180 $\mathrm{s}$. The intermittent sequence was performed five times, and started and ended with a phase of 1 $\mathrm{Gz}, 2 \mathrm{Gz}$, and $3 \mathrm{Gz}$ for $120 \mathrm{~s}$ each (Figure 1B). If participants showed abnormal vital signs and/or syncope, a physician would determine whether to terminate the protocol. This was based upon cardiovascular parameters, such as cardiac arrhythmias, including increased HR > $80 \%$ of maximal HR (220-age) for more than 10 $\mathrm{s}$, decreased HR $<50 \%$ of initial HR, or continuous decrease of HR or ABP for more than $15 \mathrm{~s}$ below initial values. Additionally, audio and video surveillance, as well as a "panic button," were available for the participant's safety during the experiment. Baseline measurement was performed during baseline rotation at 5 RPM before incremental phase 1 with the participant in a supine position, eyes closed and no one except the participant in the centrifuge room.

\section{Hemodynamic Parameters}

Electrocardiogram electrodes and a blood pressure (BP) cuff dedicated to the portable medical surveillance system (Intelivue XDS, Philips, Amsterdam, Netherlands), as well as a continuous BP device (Finometer, Portapress/TVO, Amsterdam, Netherlands), were used to monitor and record cardiovascular parameters and the vital signs of the participant. BP was continuously assessed at the participant's index finger and calculations were performed to obtain a value valid at heart level. Changes in oxyhemoglobin $\left(\mathrm{O}_{2} \mathrm{Hb}\right)$ and deoxyhemoglobin $(\mathrm{HHb})$ in the underlying tissue were recorded using a four-channel NIRS system (Oxymon MKIII, Artinis, Amsterdam, Netherlands) at the prefrontal cortex (PFC), the Musculus biceps brachii, and the Musculus gastrocnemius at a sampling frequency of 50 $\mathrm{Hz}$. The distance between the optodes and the receiver was $3.5 \mathrm{~cm}$, and this allowed a depth of penetration of approximately $2.5 \mathrm{~cm}$. The wavelengths used were $858 \mathrm{~nm}$ and $764 \mathrm{~nm}$. The NIRS optodes were fixed at the measurement points in order to avoid artifacts during the application of AG. Onset of the Gz load was assessed by an accelerometer and a trigger signal in the NIRS recording allowed for temporal alignment of the signals. $\mathrm{O}_{2} \mathrm{Hb}$ and $\mathrm{HHb}$ averages for left PFC, Musculus biceps brachii, and Musculus gastrocnemius, as well as ABP and HR for each $\mathrm{Gz}$ level were calculated and exported for later statistical analysis.

\section{Blood Collection}

BDNF, VEGF, and IGF-1 were measured from blood samples taken through a permanent venous catheter, which was inserted into each participant's left arm by a physician two hours prior to acceleration and was kept open by a $0.9 \%$ saline dilution. Immediately before the centrifugation started, and after the end of the centrifugation, $10 \mathrm{ml}$ of EDTA blood was drawn in pre-chilled vacutainers ${ }^{\circledR}$. Blood samples were centrifuged at $5000 \mathrm{RPM}$ for $10 \mathrm{~min}$ after collection in a cooled centrifuge. The plasma was divided into fractions, first stored at $-20^{\circ} \mathrm{C}$ for $8 \mathrm{~h}$, and later frozen at $-80^{\circ} \mathrm{C}$ until final analysis.

In order to quantify the serum prolactin and cortisol concentrations, an in vitro test using quantitative sandwich enzyme immunoassays (Elisa, R\&D Systems, USA) was conducted. Monoclonal antibodies specific for human BDNF, IGF-1, and VEGF were pre-coated onto a microplate. The standards and samples were pipetted into the wells and BDNF, IGF-1, or VEGF present in the sample were bound by the immobilized antibodies. After washing away any unbound substances, an enzyme-linked polyclonal antibody specific for human BDNF, IGF-1, or VEGF was added to the wells. Following a wash to remove any unbound antibody-enzyme reagent, a substrate solution was added to the wells and the color developed in proportion to the amount of proteins bound in the initial step. When the color development stopped, the intensity of the color was measured. 

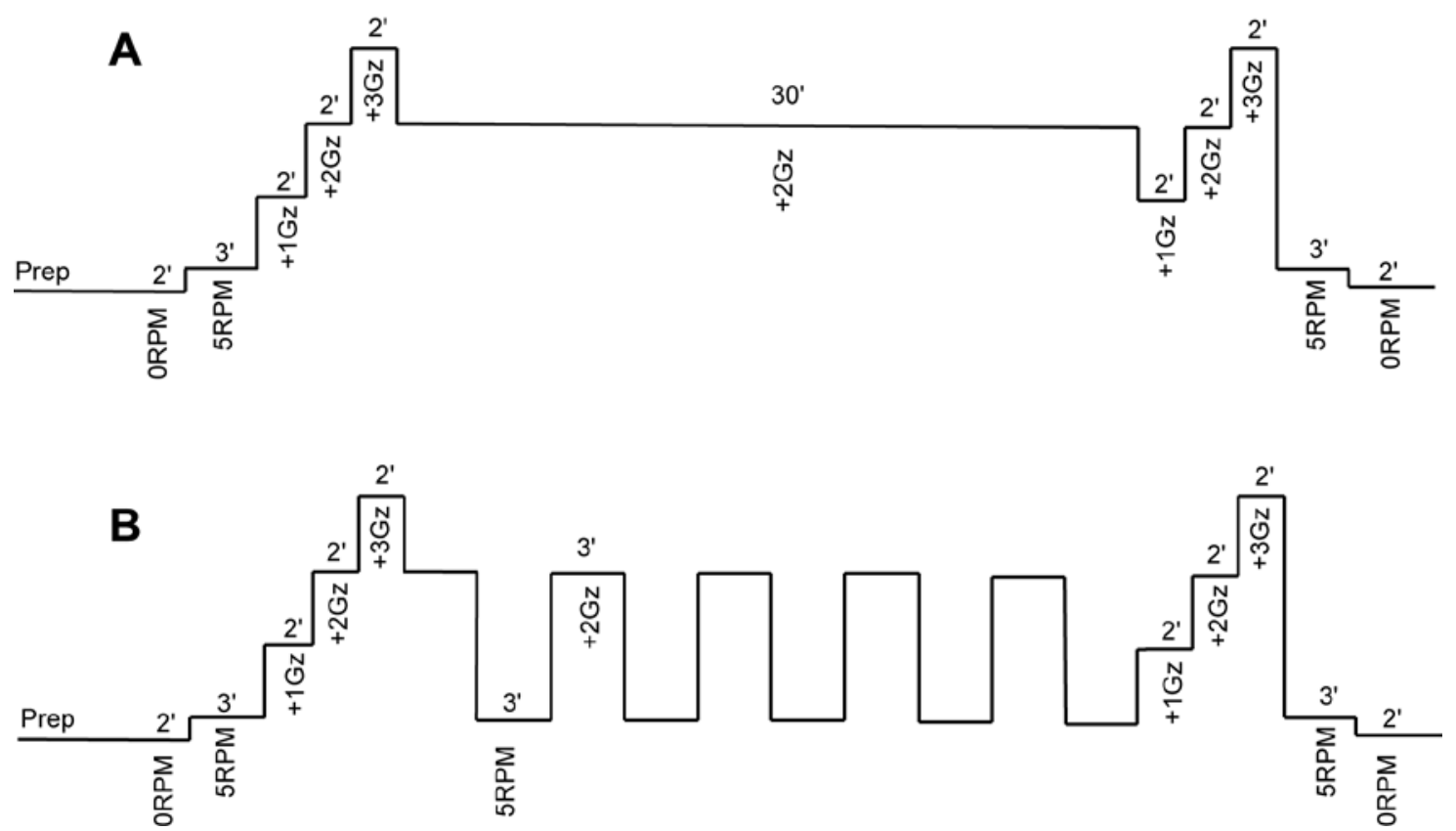

Figure 1. Experimental protocol of continuous (A) or intermittent (B) application of artificial gravity (AG). Panel (A) displays the results generated by a short arm centrifuge, illustrating periods of baseline measurement and all $\mathrm{Gz}$ accelerations (at $5 \mathrm{RPM}, 1 \mathrm{Gz}, 2 \mathrm{Gz}$, and $3 \mathrm{Gz}$ ), including the duration of each $\mathrm{Gz}$ acceleration (1', 2', and $\left.30^{\prime}\right)$. Panel $B$ displays the results generated by a short arm centrifuge, illustrating periods of baseline measurement and all Gz accelerations (at 5 RPM, 1 Gz, 2 Gz, and $3 \mathrm{Gz}$ ), including the duration of each $\mathrm{Gz}$ acceleration (1', 2', 3', and 30'). ' indicates minutes.

\section{Statistical Analysis}

Before further analysis, all data were tested for normal distribution using a one sample Kolmogorov-Smirnov test. For $\mathrm{O}_{2} \mathrm{Hb}$ and $\mathrm{HHb}$, an ANOVA test with the inter-individual factor PROTOCOL (continuous, intermittent) and the intra-individual factor TIME (course of different $\mathrm{Gz}$ levels and baseline, see Figure 1) was calculated for the three measurement points at the PFC, the Musculus biceps brachii, and the Musculus gastrocnemius. Least Significant Difference post-hoc tests were used in order to differentiate between the different $\mathrm{Gz}$ levels during the AG profiles. A second ANOVA with the inter-individual factor PROTOCOL (continuous, intermittent) and the intraindividual factor TIME (pre, post) was calculated for HR, ABP, BDNF, VEGF, and IGF-1. Significance for all statistical testing was set at $\mathrm{p}<.05$. Statistical analysis was performed using Statistica 7 (StatSoft, Tulsa, OK). Values in the text are designated as mean \pm confidence intervals $(95 \%)$.

\section{RESULTS}

\section{Hemodynamic Response}

\section{Continuous profile}

$\mathrm{O}_{2} \mathrm{Hb}$ and $\mathrm{HHb}$ in the prefrontal cortex, the Musculus biceps brachii, and the Musculus gastrocnemius were significantly affected by the changes in gravity level. Within the prefrontal cortex $\mathrm{O}_{2} \mathrm{Hb}$ decreased $\left(\mathrm{F}_{(13,117)}=26.36, \mathrm{p}<.001\right)$, whereas $\mathrm{HHb}$ increased $\left(\mathrm{F}_{(13,117)}=11.12, \mathrm{p}<.001\right)$ with increasing gravity levels. Similar effects were obtained for the Musculus biceps brachii $\left(\mathrm{O}_{2} \mathrm{Hb} \quad \mathrm{F}_{(13,}{ }_{117)}=16.49, \quad \mathrm{p}<.001 ; \quad \mathrm{HHb} \quad \mathrm{F}_{(13,}\right.$, 117) $=11.18, \quad \mathrm{p}<.001)$. At the Musculus gastrocnemius, an increase of both $\mathrm{O}_{2} \mathrm{Hb}\left(\mathrm{F}_{(13}\right.$, $\left.{ }_{117)}=1.90, \mathrm{p}<.05\right)$ and $\mathrm{HHb} \quad\left(\mathrm{F}_{(13,117)}=4.96\right.$, $\mathrm{p}<.001$ ) was observed.

Whereas HR increased with increasing gravity levels $\left(\mathrm{F}_{(13,130)}=20.34 \mathrm{p}<.001\right), \mathrm{ABP}$ only increased during the initial $2 \mathrm{G}$ and $3 \mathrm{G}$ phases $\left(\mathrm{F}_{(13,130)}=1.89, \quad \mathrm{p}<.05\right)$. Post-hoc analyses showing significant changes to baseline conditions are displayed in Figure 2 (left).

\section{Intermittent profile}

$\mathrm{O}_{2} \mathrm{Hb}$ and $\mathrm{HHB}$ values at the PFC, Musculus biceps brachii, and Musculus gastrocnemius all showed significant changes, mimicking the acceleration profile. PFC oxygenation again decreased with gravity levels $\left(\mathrm{O}_{2} \mathrm{Hb} \mathrm{F}_{(17,170)}=18.12, \mathrm{p}<.001\right)$, whereas $\mathrm{HHb}$ increased with increasing gravity levels $\left(\mathrm{F}_{(17}\right.$, $\left.{ }_{170}=5.30, \quad \mathrm{p}<.001\right)$. Similar findings were obtained for $\mathrm{O}_{2} \mathrm{Hb}\left(\mathrm{F}_{(17,170)}=9.50, \mathrm{p}<.001\right)$ and $\mathrm{HHb}\left(\mathrm{F}_{(17,170)}=5.69, \mathrm{p}<.001\right)$ at the Musculus biceps brachii. At the Musculus gastrocnemius, 


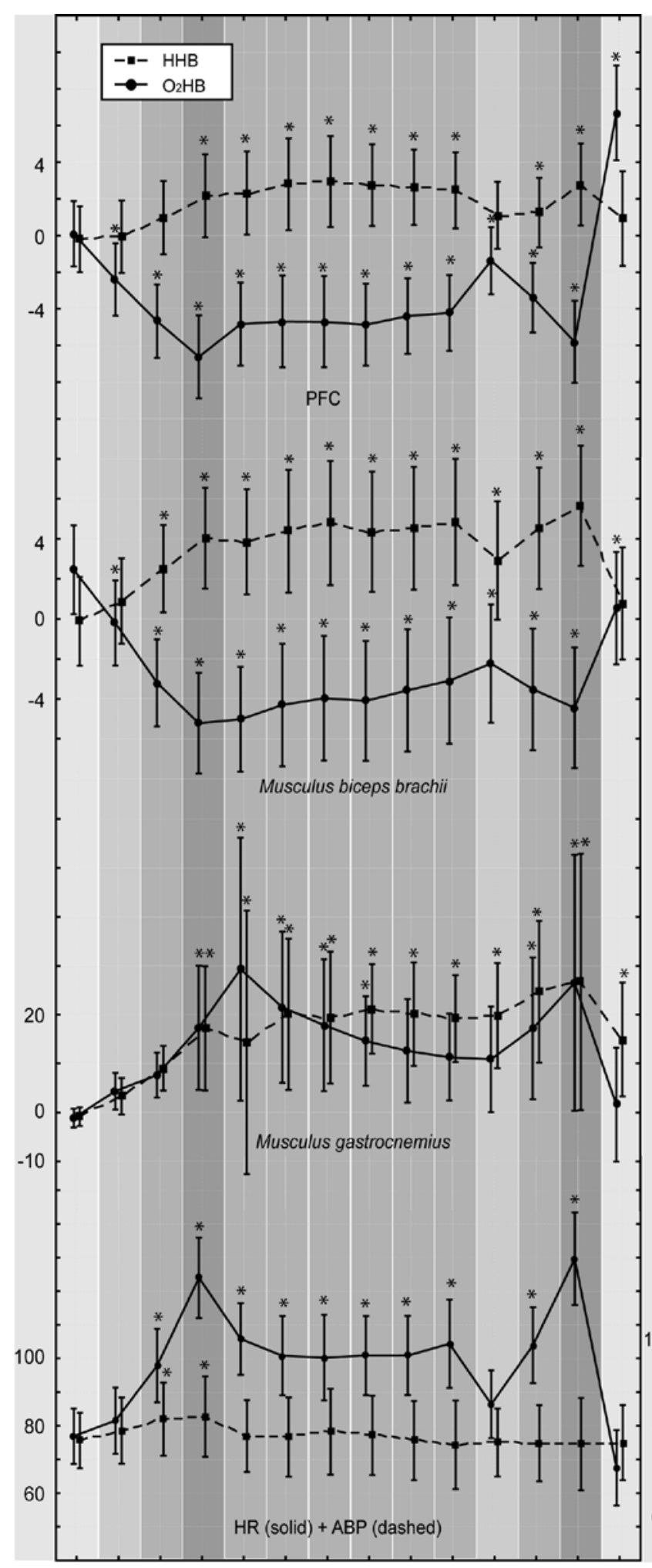

$\begin{array}{lllllllllllllll}0 G & 1 G & 2 G & 3 G & 2 G & 2 G & 2 G & 2 G & 2 G & 2 G & 1 G & 2 G & 3 G & 0 G\end{array}$

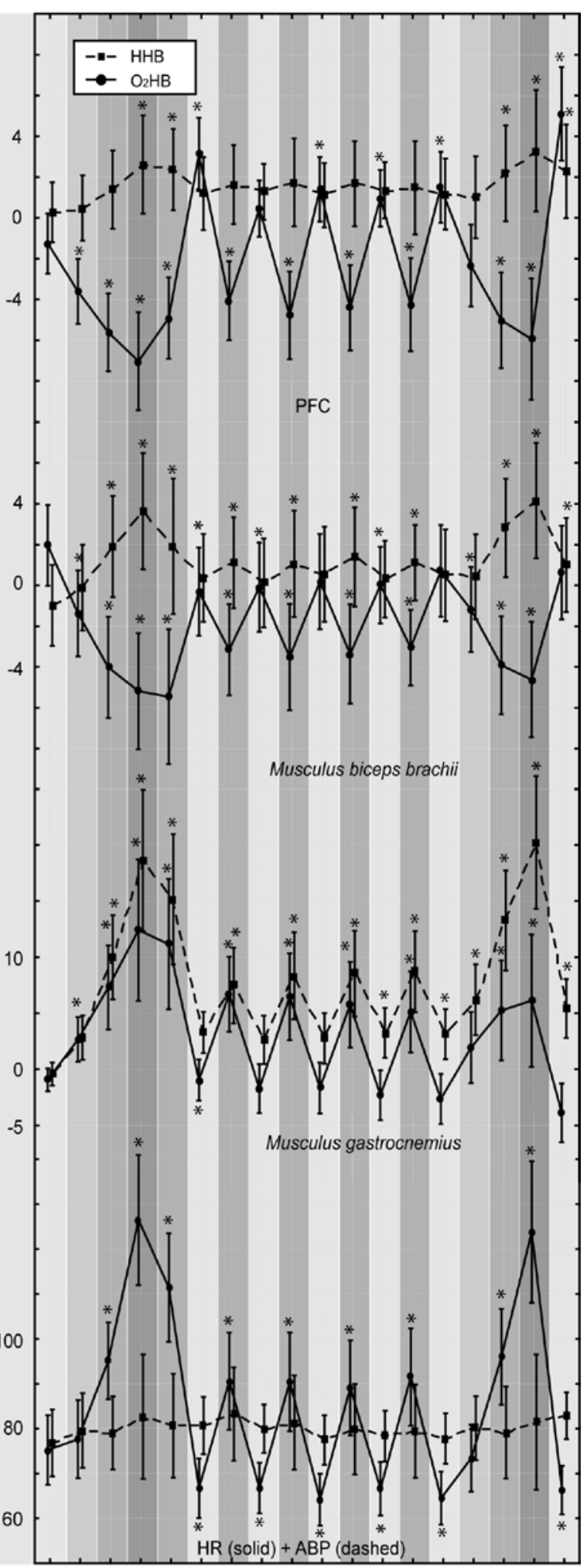

OG $1 \mathrm{G} 2 \mathrm{G} 3 \mathrm{G} 2 \mathrm{G}$ OG $2 \mathrm{G}$ OG $2 \mathrm{G}$ OG $2 \mathrm{G}$ 0G $2 \mathrm{G}$ 0G $1 \mathrm{G} 2 \mathrm{G} 3 \mathrm{G}$ OG

Figure 2. Analysis of oxygenated blood $\left(\mathrm{O}_{2} \mathrm{Hb}\right)$ and deoxygenated blood $(\mathrm{HHb})$ in response to either continuous or intermittent artificial gravity (AG) application. Means and confidence intervals (95\%) for $\mathrm{O}_{2} \mathrm{Hb}$ and $\mathrm{HHb}$ concentrations in the prefrontal cortex (PFC) are shown in the graphs on the top row, for the Musculus biceps brachii in the graphs in the second row, for the Musculus gastrocnemius in the graphs on the third row, and for heart rate (HR) and blood pressure (BP) in the graphs on the bottom row. The graphs on the left are the results from the continuous protocol, while the graphs on the right are the results from the intermittent protocol. Asterisks (*) mark differences $(p<.05)$ from baseline (0 G, 5 RPM) values. 
$\mathrm{O}_{2} \mathrm{Hb}\left(\mathrm{F}_{(17,170)}=19.02, \mathrm{p}<.001\right)$, as well as $\mathrm{HHb}$ $\left(\mathrm{F}_{(17, \quad 170)}=22.79, \quad \mathrm{p}<.001\right) \quad$ increased with increasing gravity levels. Analysis of HR also showed significant changes, mimicking the acceleration profile $\left(\mathrm{F}_{(17,170)}=75.60 \mathrm{p}<.001\right)$. There were no significant changes in $\mathrm{ABP}\left(\mathrm{F}_{(17}\right.$, ${ }_{187)}=.52 \mathrm{p}=.94$ ). Post-hoc analysis showing significant changes to baseline conditions are displayed in Figure 2 (right).

\section{Neuroendocrinological Response}

We did not observe any changes in BDNF $\left(\mathrm{F}_{(1,10)}=2.13, \mathrm{p}=.17\right)$, IGF-1 $\left(\mathrm{F}_{(1,9)}=.00, \mathrm{p}=.98\right)$, or VEGF $\left(\mathrm{F}_{(1,10)}=.18, \mathrm{p}=.68\right)$ values from pre to post centrifugation in either protocol. These results are presented in Table 1.

Table 1. Brain-derived neurotrophic factor (BDNF), insulin-like growth factor 1(IGF-1), and vascular endothelial growth factor (VEGF) values pre and post interventions.

\begin{tabular}{|c|c|c|c|c|}
\hline & \multicolumn{2}{|c|}{ AG constant [pg/ml $]$} & \multicolumn{2}{c|}{ AG intermittent [pg/ml] } \\
\hline & Pre & Post & Pre & Post \\
\hline $\begin{array}{c}\text { BDNF } \\
\mathrm{n}=11\end{array}$ & $12212,66 \pm 8716,77$ & $10028,43 \pm 10637,02$ & $9912,01 \pm 10748,13$ & $12195,94 \pm 11949,15$ \\
\hline $\begin{array}{c}\text { IGF-1 } \\
\mathrm{n}=10\end{array}$ & $139,31 \pm 68,64$ & $144,48 \pm 74,03$ & $137,99 \pm 60,75$ & $143,53 \pm 69,55$ \\
\hline $\begin{array}{c}\text { VEGF } \\
\mathrm{n}=10\end{array}$ & $174,77 \pm 146,25$ & $159,61 \pm 169,65$ & $186,29 \pm 166,47$ & $180,22 \pm 181,45$ \\
\hline
\end{tabular}

Data is presented as mean \pm standard deviation.

\section{DISCUSSION}

One aim of this study was to describe the hemodynamic and tissue oxygenation responses to continuous and intermittent periods of $\mathrm{AG}$ compared to what would be expected during a moderate bout of exercise. This study was conducted in a SAHC, with the aim of investigating the use of $A G$ as a potential countermeasure to physiological deconditioning during long-duration human spaceflight. The results suggest that both the continuous and the intermittent AG protocols induced an increase in Musculus gastrocnemius $\mathrm{O}_{2} \mathrm{Hb}$ and $\mathrm{HHb}$, most likely representing an increased pooling of blood in the lower limbs. Considering a simple shift of blood away from the head and upper body during centrifugation, one might assume there would be a decrement in both $\mathrm{O}_{2} \mathrm{Hb}$ and $\mathrm{HHb}$ concentrations. Instead, as we have proposed previously (Schneider et al., 2013), there was a reduction in arterial flow to the brain and Musculus biceps brachii muscle during the continuous application of $2 \mathrm{Gz}$ and $3 \mathrm{Gz}$, evidenced by the reduction in $\mathrm{O}_{2} \mathrm{Hb}$ in these regions. The reduction in flow to the upper body can be explained by a reduced pressure gradient for flow towards the upper body (against the AG) (Schneider et al., 2013), and as a result, less oxygenated blood reaches the brain and the biceps. There were no apparent ill effects of this response; participants did not demonstrate any signs of syncope and BP remained relatively constant. The rise in $\mathrm{HHb}$ at the PFC and biceps during the constant AG protocol is consistent with a decreased tissue oxygenation index observed during hypergravity (Kurihara et al., 2007; Schneider et al., 2013) and during head-up tilt (Kurihara et al., 2003). This may represent a relative increase in oxygen extraction from the circulation to compensate for the lower arterial flow to the upper body. It is also plausible that oxygen demand, and therefore extraction, is higher during hypergravity due to increased physical demands, as reflected by the observed increase in HR.

During the intermittent protocol, there were rapid and large increases in $\mathrm{O}_{2} \mathrm{Hb}$ at the $\mathrm{PFC}$ and the biceps during the periods of reduced centrifugal forces in the head-to-toe axis. Consistent with the earlier interpretation, this likely reflects a transient restoration of the pressure gradient towards the upper body, and an increase in blood flow to the head and arms. In some cases, this increase in $\mathrm{O}_{2} \mathrm{Hb}$ exceeded baseline values, particularly at the PFC, which may represent a reactive hyperaemia response to the periods of AG. These transient increases in 
$\mathrm{O}_{2} \mathrm{Hb}$ (and flow) might also be mediated by central hemodynamic changes, whereby an abrupt increase in venous return to the right atria with a reduction in gravity $(0 \mathrm{G})$ contributes to an increase in stroke volume and driving pressure for blood flow. The observed reduction in HR during the periods of $0 \mathrm{G}$ to levels below baseline might partly be a compensatory response to maintain cardiac output. Interestingly, these periods of normal gravity and restored upper-body blood flow during the intermittent protocol were associated with a slight $(\mathrm{p}<.05)$ attenuation of rise in $\mathrm{HHb}$, particularly at the Musculus biceps brachii $(\mathrm{p}<.05)$, compared with the continuous protocol. This may again reflect a reduced metabolic demand and oxygen use, and/or a reduced local oxygen extraction during periods when arterial flow is restored. Another major reason for hemodynamic changes after the reduction of centrifugal force and the gradient of the centrifugal force might be an increment of flowthrough carotid arteries and a decrement of deoxygenized blood in the brain, caused by blood being pulled from the brain by gravitational forces.

We recently suggested (Schneider et al., 2013) that a reduction in gravity allows more oxygenated blood with each pulse wave to be directed to the low-resistance vessels of the head. This leads to a significant increment in $\mathrm{O}_{2} \mathrm{Hb}$ concentration. In contrast to that, the $\mathrm{HHb}$ concentration decreases only slightly, since no additional gravitational forces are pulling blood out of the brain, and the oxygen consumption of the brain remains stable throughout the application of AG (Schneider et al., 2013).

The absence of a significant change in BP during the application of AG was somewhat unexpected given the typical alterations in autonomic activity with changes in gravitational force (Linnarsson et al., 2015; Migeotte et al., 2009). The stable BP might be an adaptation, such that there is a sequestration of blood in capacitance veins in the lower peripheral extremities, demonstrated using computer-based simulations by Summers and colleagues (Summers et al., 2010). Alternatively, a gradual venous pooling in the peripheral extremities might cause a reflex myogenic constriction, including the blood vessels in the index finger, which could have an impact on the continuous assessment of BP.

Although AG at a tolerable level $(<2 \mathrm{Gz})$ is related to an increase in HR, this increase is far below what is experienced during moderate exercise. At this level of AG, no changes in ABP were detected. The positive effect of exercise on cardiovascular health is based on the physiological stress caused by exercise. Our data suggests that moderate doses of AG at or below $2 \mathrm{Gz}$ are not sufficient to create a relevant physiological stressor for cardiovascular training. The same is true for the release of neurotrophic factors, which are not affected by either of the AG protocols (Table 1). Several studies on the effects of exercise on the release of neurotrophic factors showed that only high intensity exercise is related to an increase in neurotrophic factors (Rojas Vega et al., 2006). As shown by Schiffer et al. (2011) using lactate infusion, the driving factor for a release of neurotrophic factors seems to be lactate, which in young healthy persons, is released only at exercise intensities that induce a HR of 180 -age (Hollmann et al., 2012; McArdle et al., 2015).

With respect to the data in the present study, much higher values of $\mathrm{AG}=3 \mathrm{Gz}$ (Figure 2) seem necessary to increase HR to a level similar to moderate physical activity, which is around 180-age. Nevertheless, this level is barely tolerated by the individual, at least not for a long duration. In order to promote vascular adaptations that offset the negative effects of spaceflight, an intermittent protocol with $\mathrm{Gz}=3$ Gz would be a better choice compared to a constant protocol; intermittent AG might lead to increases in blood flow and shear stress, which would in turn promote maintenance or improvement of vascular function (e.g., endothelial function), or even vascular adaptations such as an increase in vessel size.

It should be acknowledged that the study is missing measures of central hemodynamics and blood flow. NIRS has the advantage of being applicable in extreme situations, but interpretation of findings needs to be validated by, for example, a Doppler Ultrasound. This would also clarify possible interference of scalp and/or skull perfusion and blood flow at the PFC. A recent study by Ogawa et al. (2016) showed a decrease in cerebral blood flow velocity when exposed to artificial gravity, similar to the $\mathrm{O}_{2} \mathrm{Hb}$ values in the PFC described in the present study.

\section{ACKNOWLEDGEMENTS}

We would like to express our gratitude to all individuals who participated in this study. We would also like to acknowledgment the SAHC staff of the German Aerospace Centre, Cologne, as well as Sebastian Dern, Amrei 
Jacubowski, and Petra Wollseiffen for their help with data collection.

\section{CONFLICT OF INTEREST}

This study was funded by a research grant from the German Aerospace Centre (DLR, Grant 50WB1161). The authors have nothing to disclose.

\section{AUTHOR CONTRIBUTIONS}

VSZ, TV, AJ, and VA carried out the studies and participated in the analysis. SS and CDA drafted and finalized the manuscript, and $\mathrm{HC}$ edited the manuscript. HKS conceived of the study, and participated in its design and coordination, and helped to draft the manuscript. All authors have read and approved the final version of the manuscript and agree with the order of presentation of the authors.

\section{REFERENCES}

Barr MJ, Gabbett TJ, Newton RU, Sheppard JM (2015) Effect of 8 days of a hypergravity condition on the sprinting speed and lowerbody power of elite rugby players. Journal of Strength and Conditioning Research 29: 722-729

Chouker A, Feuerecker B, Matzel S, Kaufmann I, Strewe C, Hoerl M, Schelling G, Feuerecker M (2013) Psychoneuroendocrine alterations during 5 days of head-down tilt bed rest and artificial gravity interventions. European Journal of Applied Physiology 113: 2057-2065

Clement G, Bukley A (2007) Artificial Gravity, Berlin: Springer

Clement G, Pavy-Le Traon A (2004) Centrifugation as a countermeasure during actual and simulated microgravity: a review. European Journal of Applied Physiology 92: 235-248

Clement GR, Bukley AP, Paloski WH (2015) Artificial gravity as a countermeasure for mitigating physiological deconditioning during long-duration space missions. Frontiers in Systems Neuroscience 9: 92

Dern S, Vogt T, Abeln V, Struder HK, Schneider S (2014) Psychophysiological responses of artificial gravity exposure to humans. European Journal of Applied Physiolology 114: 2061-2071

Evans JM, Ribeiro LC, Moore FB, Wang S, Zhang Q, Kostas V, Ferguson CR, Serrador J, Falvo M, Stenger MB, Goswami N, Rask JC, Smith JD, Knapp CF (2015)
Hypovolemic men and women regulate blood pressure differently following exposure to artificial gravity. European Journal of Applied Physiology 115: 26312640

Goswami N, Evans J, Schneider S, von der Wiesche M, Mulder E, Rossler A, Hinghofer-Szalkay H, Blaber AP (2015) Effects of individualized centrifugation training on orthostatic tolerance in men and women. PloS One 10: 0125780

Hollmann W, Knigge K, Knicker A, Strüder HK (2012) Methods for measurement of physical fitness and recommendations in studies on humans. In Functional Neuroimaging in Exercise and Sport Sciences, Boecker H, Hillman CH, Scheef L, HS (eds), pp 79-107. New York, Heidelberg, Dordrecht, London: Springer Science+Business Media

Knaepen K, Goekint M, Heyman EM, Meeusen R (2010) Neuroplasticity - exercise-induced response of peripheral brain-derived neurotrophic factor: a systematic review of experimental studies in human subjects. Sports Medicine 40: 765-801

Kurihara K, Kikukawa A, Kobayashi A (2003) Cerebral oxygenation monitor during headup and -down tilt using near-infrared spatially resolved spectroscopy. Clinical Physiology and Functional Imaging 23: 177-181

Kurihara K, Kikukawa A, Kobayashi A, Nakadate T (2007) Frontal cortical oxygenation changes during gravity-induced loss of consciousness in humans: a nearinfrared spatially resolved spectroscopic study. Journal of Applied Physiology (1985) 103: $1326-1331$

Linnarsson D, Hughson RL, Fraser KS, Clement G, Karlsson LL, Mulder E, Paloski WH, Rittweger J, Wuyts FL, Zange J (2015) Effects of an artificial gravity countermeasure on orthostatic tolerance, blood volumes and aerobic power after short-term bed rest (BR-AG1). Journal of Applied Physiology (1985) 118: 29-35

Lista I, Sorrentino G (2010) Biological mechanisms of physical activity in preventing cognitive decline. Cellular and Molecular Neurobiology 30: 493-503

McArdle WD, Katch FI, Katch VL (2015) Exercise Physiology, Baltimore: Wolters Kluwer Health

Migeotte PF, Pattyn N, Vanspauwen R, Neyt X, Acheroy M, Van de Heyning P, Wuyts 
FL (2009) Respiratory sinus arrhythmia on the ESA-short-arm human centrifuge. IEEE Engineering in Medicine and Biology Magazine 28: 86-91

Ogawa Y, Yanagida R, Ueda K, Aoki K, Iwasaki K (2016) The relationship between widespread changes in gravity and cerebral blood flow. Environmental Health and Preventative Medicine 21: 186-192

Pavy-Le Traon A, Heer M, Narici MV, Rittweger J, Vernikos J (2007) From space to Earth: advances in human physiology from 20 years of bed rest studies (19862006). European Journal of Applied Physiology 101: 143-194

Richter C, Braunstein B, Winnard A, Nasser M, Weber T (2017) Human biomechanical and cardiopulmonary responses to partial gravity - a systematic review. Frontiers in Physiology 8: 583

Rojas Vega S, Knicker A, Hollmann W, Bloch W, Struder HK (2010) Effect of resistance exercise on serum levels of growth factors in humans. Hormone and Metabolic Research 42: $982-986$

Rojas Vega S, Struder HK, Vera Wahrmann B, Schmidt A, Bloch W, Hollmann W (2006) Acute BDNF and cortisol response to low intensity exercise and following ramp incremental exercise to exhaustion in humans. Brain Research 1121: 59-65

Schiffer T, Schulte S, Hollmann W, Bloch W, Struder HK (2009) Effects of strength and endurance training on brain-derived neurotrophic factor and insulin-like growth factor 1 in humans. Hormone and Metabolic Research 41: 250-254
Schiffer T, Schulte S, Sperlich B, Achtzehn S, Fricke H, Struder HK (2011) Lactate infusion at rest increases BDNF blood concentration in humans. Neuroscience Letters 488: 234-237

Schneider S (ed) (2016) Exercise in Space - A Holistic Approach for the Benefit of Human Health on Earth, Switzerland: Springer

Schneider S, Abeln V, Askew CD, Vogt T, Hoffmann U, Denise P, Struder HK (2013) Changes in cerebral oxygenation during parabolic flight. European Journal of Applied Physiology 113: 1617-1623

Summers RL, Platts S, Myers JG, Coleman TG (2010) Theoretical analysis of the mechanisms of a gender differentiation in the propensity for orthostatic intolerance after spaceflight. Theoretical Biology and Medical Modelling 7: 8

Tanaka K, Nishimura N, Kawai Y (2017) Adaptation to microgravity, deconditioning, and countermeasures. Journal of Physiological Sciences 67: 271-281

Waki H, Katahira K, Yamasaki M, Nagayama T, Katsuda S, Wago H, Okouchi T, H OI, Miyake M, Miyamoto Y, Shimizu T (2005) Effects of spaceflight on postnatal development of arterial baroreceptor reflex in rats. Acta Physiologica Scandinavica 184: 17-26

Zhang Q, Evans JM, Stenger MB, Moore FB, Knapp CF (2017) Autonomic cardiovascular responses to orthostatic stress after a short artificial gravity exposure. Aerospace Medicine and Human Performance 88: 827833 